\title{
Decrease in asthma exacerbation prevalence during the Covid-19 lockdown in a moderate-severe asthma cohort
}

\author{
Geertje de Boer ${ }^{1}$, Gert-Jan Braunstahl ${ }^{1}$, Rudi Hendriks ${ }^{2}$, and Gerdien Tramper ${ }^{1}$ \\ ${ }^{1}$ Franciscus Gasthuis en Vlietland \\ ${ }^{2}$ Erasmus MC
}

July 7,2020

\section{Decrease in asthma exacerbation prevalence during the Covid-19 lockdown in a moderate- severe asthma cohort}

To the editor,

Following the recent Covid-19 lockdown, in Canada a reduction in emergency healthcare visits was reported (1). Infectious diseases were less often diagnosed, probably due to social distancing and increased hygiene measures. However, also fear of acquiring Covid-19 infection at medical facilities might have led to a decrease in visits $(1,2)$.

Recent studies indicated that controlled asthma is not a risk factor for severe disease in the current Covid19 pandemic (2-5). Entry of the SARS-CoV2 virus is mediated by the receptor for angiotensin-converting enzyme-2 (ACE2). The expression of ACE2 seems to be modulated by type 2 inflammation, which is present in the majority of asthma patients and might thus confer protection against severe Covid-19 symptoms (3, $4,6)$.

Social distancing is known to reduce the spread of seasonal influenza and viral respiratory tract infections (RTI) (2, 7). We hypothesize that this reduction in viral transmission during the Covid-19 lockdown is responsible for a decrease in asthma exacerbations (AE), which are often elicited by viral RTI. Therefore, we analyzed in a cohort of patients with moderate-severe asthma and recurrent AE the effect of the Covid-19 lockdown on the frequency of severe AE requiring oral corticosteroids and health care avoidance.

This study comprises a follow-up from the recently completed Breathe study (NL5752) that included adult asthma patients on step GINA 3-4 treatment with recurrent exacerbations. Control individuals without asthma from the cross-sectional Grandma study (NCT03278561) were included as a reference population (see: online supplementary material).

Participants were invited to fill out a short survey by e-mail or post (see: online supplementary material), including an asthma control questionnaire (ACQ), an asthma quality of life questionnaire (AQLQ) and a hospital anxiety and depression scale (HADS), as well as questions on exacerbation frequency and care avoidance between April $12^{\text {th }}$ and June $1^{\text {st }}$ 2020. Two reminders were sent out. Exacerbation frequencies until July $1^{\text {st }}$ were verified with the hospitals' and general practitioner's medical records and pharmacy. Covid-19 restrictions in The Netherlands started mid-March and were partly lifted since early June 2020 in a stepwise fashion.

Clinical parameters from the original study visits were used for baseline characteristics. Primary outcome was the difference in number of AE between the second quarters (Q2) of 2020 and the three years prior to the Covid-19 pandemic. Only severe AE needing oral corticosteroids and/or antibiotics were included. 
Secondary outcomes were ACQ, AQLQ, HADS, health care avoidance and fear of COVID-19. ACQ and AQLQ were compared with April 2019.

Differences between asthma and controls were evaluated with the chi-square-test, Student's-t-test or the Mann-Whitney-U-test depending on the variable and its distribution. Differences between time points were compared with a Friedman test, Bonferroni correction was applied for the Wilcoxon-signed-rank-test where a p-value was set at 0.0083 . Data is shown in median $\left[25^{\text {th }}-75^{\text {th }}\right]$ or mean values \pm standard deviation. A p-value $<0.05$ was deemed significant. Statistical analyses were conducted with SPSS 26.0.

Out of 94 invited participants ( 67 asthma; 27 controls), 67 participants ( $71 \%)$ were included. No significant differences were observed in age, BMI, comorbidities and other demographics such as prevalence of proven Covid-19 (Table 1).

In quarter $2(\mathrm{Q} 2)$ of 2020 mean exacerbation frequency per patient was significantly lower $\left(\chi^{2}(3)=9.91\right.$, $\mathrm{p}=0.019)$, compared to Q2 of $2017(Z=-2.67, \mathrm{p}=0.008), 2018(Z=-2.50, \mathrm{p}=0.012)$ and $2019(Z=-3.26$, $\mathrm{p}=0.001)$. No difference in mean exacerbation frequency was seen between Q1 of 2017, 2018, 2019 and 2020. Mean exacerbation frequency per patient per quarter did not differ between the years 2017, 2018 and 2019 (Figure 1). AE in 2020 were not related to positive SARS-CoV2 polymerase chain reaction or hospitalizations. Asthma control and AQLQ in Q2 of 2020 was comparable with April 2019.

Asthma patients were more likely to avoid (38.8\%; controls, $0.0 \%, \mathrm{p}<0.01)$ or delay $(24.5 \%$; controls, $0.0 \%$, $\mathrm{p}=0.02$ ) essential medical visits due to fear of SARS-CoV2 infection at those facilities. In case of $\mathrm{AE}$, e-consults were used by most patients $(83.3 \%)$.

Fear was objectified by a clinically relevant higher HADS score reflecting a possible anxiety or depression disorder in asthma patients compared to controls $(8.00$ (5.00-12.50) vs. $4.00(1.00-7.00), \mathrm{p}<0.01)$.

In this study, we identified a decrease in AE during the Covid-19 lockdown measures in patients with moderate-severe asthma. We expect social distancing to be responsible for this decrease. Asthma patients experienced more fear of SARS-CoV2 infection compared with controls. Nevertheless, fear of acquiring a SARS-CoV2 infection at medical facilities did not lead to missed AE or seriously delayed care for an AE because of e-consulting possibilities.

Recent studies indicate that asthma is not a risk factor for severe disease in the current Covid-19 pandemic and that type 2 inflammation might be protective (2-6). Thus, an increased fear of Covid-19 experienced by asthma patients seems irrational. Following the warnings from the Dutch National Institute for Public Health and reports in the media, however, it is conceivable that asthma patients considered themselves at risk (3).

Although the sample size in our study was low, it is of note that frequencies of AE during the Covid-19 pandemic could be compared with baseline data acquired by quarterly monitoring of AE over the past three years.

While asthma patients would rather delay or avoid visits to medical care facilities, they did reach out for medical assistance if needed via e-consult, thereby preventing delay of essential medical care.

In conclusion, we found a significantly reduced AE frequency during Covid-19 social distancing measures compared to previous years. However, asthma patients also showed more general anxiety and anxiety towards acquiring Covid-19 infection. Because the risk for acquiring Covid-19 infection will be present for a yet unknown period, it is important to accurately inform asthma patients about the real risks. This would contribute to a reduction of unnecessary anxiety and may encourage them to contact their practitioner by e-consults.

Figure / Table legends:

Table 1: Participant demographics

Data shown in mean $\pm S D$ and median [25th-75th] or absolute counts $N(\%)$. 
Figure 1: Asthma exacerbations in the years 2017-2020

1. Mean number of exacerbations per patient per quarter per year; * =statistically significant from Q2 in other years. [?] undefined

2. Number of patients with 0, 1, >1 exacerbation in Q2 per year

\section{References}

1. Bres Bullrich M, Fridman S, Mandzia JL, Mai LM, Khaw A, Vargas Gonzalez JC, et al. COVID-19: Stroke Admissions, Emergency Department Visits, and Prevention Clinic Referrals. Can J Neurol Sci. 2020:1-10.

2. Copeland DL, Basurto-Davila R, Chung W, Kurian A, Fishbein DB, Szymanowski P, et al. Effectiveness of a school district closure for pandemic influenza A (H1N1) on acute respiratory illnesses in the community: a natural experiment. Clin Infect Dis. 2013;56(4):509-16.

3. Jackson DJ, Busse WW, Bacharier LB, Kattan M, O'Connor GT, Wood RA, et al. Association of respiratory allergy, asthma, and expression of the SARS-CoV-2 receptor ACE2. J Allergy Clin Immunol. 2020.

4. Ziegler CGK, Allon SJ, Nyquist SK, Mbano IM, Miao VN, Tzouanas CN, et al. SARS-CoV-2 Receptor ACE2 Is an Interferon-Stimulated Gene in Human Airway Epithelial Cells and Is Detected in Specific Cell Subsets across Tissues. Cell. 2020;181(5):1016-35 e19.

5. Riggioni C, Comberiati P, Giovannini M, Agache I, Akdis M, Alves-Correia M, et al. A compendium answering 150 questions on COVID-19 and SARS-CoV-2. Allergy. 2020.

6. Carli G, Cecchi L, Stebbing J, Parronchi P, Farsi A. Is asthma protective against COVID-19? Allergy. 2020 .

7. Noh JY, Seong H, Yoon JG, Song JY, Cheong HJ, Kim WJ. Social Distancing against COVID-19: Implication for the Control of Influenza. J Korean Med Sci. 2020;35(19):e182.

Geertje M. de Boer ${ }^{1,2}$, Gert-Jan Braunstahl ${ }^{1,2}$, Rudi W. Hendriks ${ }^{2}$, Gerdien A. Tramper-Stranders ${ }^{3}$

1. Department of Pulmonology, Franciscus Gasthuis \& Vlietland, Rotterdam, The Netherlands

2. Department of Pulmonology, Erasmus MC, Rotterdam, The Netherlands

3. Department of Paediatrics, Franciscus Gasthuis \& Vlietland, Rotterdam, The Netherlands

\section{Acknowledgements}

We thank dr. B. Boxma-de Klerk for her advice on statistical matters.

\section{Author statement}

GMB conceived the idea for this extra follow-up and set up the study under close supervision of GB and GT. GT conceived the idea of the original cohort study. GMB wrote the manuscript and all authors reviewed it during different phases. GMB, GT and RWH constructed figure 1. All authors approved the final version of the manuscript.

\section{Conflict of interest}

Gerdien A. Tramper reports an ISS grant from OM-Pharma for an affiliated project.

\section{Hosted file}

Table 1.docx available at https://authorea.com/users/339358/articles/465595-decrease-inasthma-exacerbation-prevalence-during-the-covid-19-lockdown-in-a-moderate-severe-asthmacohort 

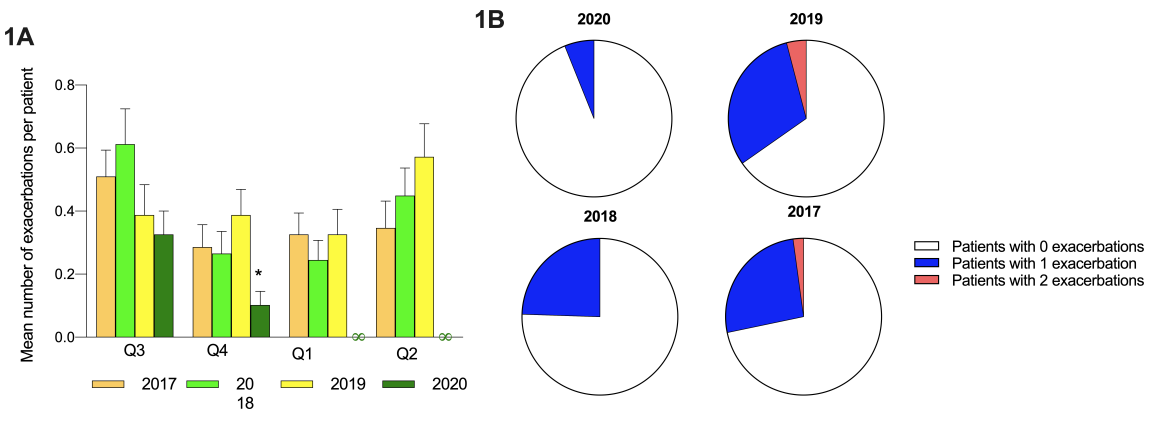